\title{
Research Report on Fan Blades Intelligent Cleaning Robot Hao Zheng ${ }^{1}$, Yicun Gong ${ }^{1}$, Ying Long ${ }^{1}, \mathrm{Si} \mathrm{Li}^{1}$ \\ ${ }^{1}$ North China Electric Power University, Beijing, China, 102206 \\ hunter2011@foxmail.com
}

Keywords: Intelligent; Wind Power; Energy Saving; Security

\begin{abstract}
As China's wind farms increase rapidly and wind power installed capacity is growing, cleaning attendant wind turbine maintenance problems will become increasingly prominent. Most fans installed in harsh environments, high altitude, complex climate, while the leaf and work in high-altitude, all-weather conditions, all kinds of media all the time in the erosion of the blade, the failure rate of about more than one-third. Regular inspection and maintenance, early detection, take immediate measures to solve the problem in the bud is to avoid accidents, reduce risk, the most effective way to stabilize farm income. Based on the difficulties currently have in the blade cleaning technology, intelligent fan blade cleaning device we studied can automatically adsorbed on work surface, cleaning blade operations independently, allowing technicians freed from the heavy operation, as far as possible to avoid many risk factors in high-altitude operations and those have practical significance and feasibility.
\end{abstract}

\section{Introduction}

China Wind Power Prospects. China's wind resources are very rich. According to National Weather Service data, our ground 10m high wind resources total reserves of about 226 million kW. Offshore wind energy can be developed and utilized reserves of 750 million $\mathrm{kW}$. Taking into account the actual use of land and other factors that can actually take advantage of the recent preliminary estimate of onshore wind energy reserves of about 800 million $\mathrm{kW}$, the actual use of offshore wind energy reserves of about 200 million $\mathrm{kW}$, a total of about one billion $\mathrm{kW}$.

Demand and the Importance of Cleaning Blade. Wind turbine blades on the windward side (commonly known as the spines, the leading edge) is the main power source generators, the spines of smoothness is a major factor in determining the speed of the fan. Leaf width ridge itself has generated resistance in the air, if you have to because of dust accumulation, producing blisters, cracks and breakage, resistance can be imagined. According to reports, downtime rate because of problems caused by the blades of about $30 \%$ or more. Long-running blade surface will accumulate a lot of dirt, the surface of the tip and cut wind surface dirt particularly serious. These soils are mostly metal particles in the air and dust, if not promptly treated can cause weight imbalance of the blade, and the blade counterweight disorders can lead to the spindle drive is not in a plane, spindle rotation is transmitted to an irregular transmission, irregular friction gear gearbox gears lead to increased wear, resulting in a lot of heat, spontaneous combustion may produce a serious explosion. Occasional cleaning and maintenance of long-term in a state where the blade shortened life expectancy, and may result in up to $20 \%$ of the amount of power loss, the impact of the economic benefits of the safe operation of wind turbine farms.

Every fan normal periodic maintenance and repair cost of only single wind turbine generating revenue of around 3\%, pre-maintenance effort in exchange for a small amount of stable power generation revenue, the losses and risks minimized.

Blade Cleaning Technology Status Abroad. Currently, the market is not able to implement intelligent automatic control of fan blade cleaning robots, but in practice, the cleaning blade maintenance through technical personnel, completed in aerial work. Staff use lifting equipment, or use of air ropes (Spider-Man) maintenance and other forms of aerial blade repair. Low-tech operation, the risk factor is high and inefficient. 


\section{Research Purposes}

In order to solve the current difficulties fan blades of the cleaning process, the present work intends to use machines instead of doing the fan blades for cleaning, reduce manual cleaning fan blades practice risk and investment. Through the intelligent fan blade surface cleaning, the stains on the blades to reduce resistance when rotating fan blades in power generation, can improve the efficiency of wind turbines, and extend the life of wind turbines, energy savings, while reducing manpower during cleaning operations the risk factor and economic investment.

\section{Practical Significance and Market Value of Products}

The products are used in wind turbine blades intelligent automatic cleaning, can greatly reduce the labor involved in cleaning business is one of the fan blades new breakthrough. Use of the product can reduce the stain resistance due to the presence of the fan blades rotate power generation to ensure that the weight of the fan blades than three and ability to catch the wind in a relatively balanced state, thus reduce the fan blades during operation of the tremor affect the host component life, improve the efficiency of wind turbines, energy savings, while reducing the risk of manual operation and related processes in the human and material resources and improve overall economic efficiency of wind power plants.

The product is easy to operate, easy to repair and maintain, through the product to adjust the size of the relevant procedures or specifications, to meet the cleaning needs of different types of wind turbine blades. After its promotion in the market, it can be used for various different types of wind turbine blades wind farm cleaning, changing the current status of the high risk of doing the cleaning process of the existence of high investment. In addition, the product can also be performed on a flat vertical or horizontal surface cleaning work, a wide range, the future will have broad market prospects and considerable economic benefits.

\section{Development Process}

Cleaning the Fan Blades to Determine the State. China is now generally clean fan blades approach is when the fan stops working, the cleaning blade to be adjusted to a vertical position by a manually operated lift height spider, elevating work platform on the blade clean. But there are a lot of dangerous high-altitude operations, human actions when cleaning the blade and lifting platforms have more inconvenience. Therefore, we consider to replace manpower with automated cleaning apparatus for cleaning blade, cleaning device can automatically carry out all-round along the blade surface clean and efficient. We still choose the fan blades raised vertically in order to artificially regulate the safety rope through the cleaning position and prevents it falls from a height, while in a horizontal direction of the blade to avoid cleaning the safety rope in the event of an unexpected situation when compared to the serious swing, with greater security.

Fan Blade Design of Intelligent Cleaning Robot. In determining the state of the cleaning fan blade (blade in a vertical direction), the for the realization of intelligent automatic cleaning purposes, we will device is designed for two parts: the control box and cleaner. Control box fixed to the root of the blade, including the PLC (programmable logic controller), transformers, water tanks, electric valves, etc., for controlling the entire cleaning robot as well as the cleaning device to provide cleaning liquid; cleaner is bonded to the surface of the blade and can be freely mobile, including high-speed vacuum centrifugal fan, track wheels, motor-driven brush, plastic, stainless steel stents and other components, for the full range of blade clean. Control box and cleaner by the cable, pipe connection as a whole

Initial Plant Design. Control box using lightweight plastic material, including PLC (programmable logic controller) and the transformer (24V DC to $220 \mathrm{~V}$ AC into), located in the upper part of the tank for controlling the switching of the entire apparatus, electric valve on the tank cleaning travel, start and stop the motor and other devices, it is the hub of the entire device. PLC 
directly regulate the operation, saving manpower and automatic cleaning. Tank same as the plastic material, which bloom good deployment cleaning fluid, cleaning fluid in the cleaning process from the electric outlet valve on the tank outflow pipe via a fixed port in the side of the spray cleaner to work sites, used for brush cleaning blade. Safety clasp one end fixed to the blade root, and the other end is fixed to the cleaning, the middle safety rope length can be adjusted to ensure that the cleaning blade surface in the process of moving, not because of adsorption is not solid but loose in improving the cleaning work when the safety factor.

As the wind turbine blades for the glass fiber material, not magnetic, can not control the use of magnets in principle cleaner is bonded to the surface of the blade, so we refer Climbing car window and Kesi Wo 8 Po works in cleaning the bottom of the center position installation of high-speed rotation speed vacuum centrifugal fans, centrifugal fans can be cleaned with the air gap between the fan blades detached, a negative pressure environment, under the pressure difference in the cleaning blade is adsorbed on the surface. The left and right sides of the high-speed vacuum centrifugal fan installation of two silicone track wheels, track wheel internally by motor control, not only can drive back and forth to clean the road, you can also adjust the forward direction of the washer, the washer can reach a full range of movement of the blade surface purposes. Silicone track wheels with a certain degree of flexibility, the area of contact with the fan blade is larger, you can better fit on the blade surface. Cleaning the housing of the same plastic material as the light from the handle of the plastic housing of the respective motor and the control box is connected to power lines. Cleaner top with stainless steel bracket for fixing the outer additional cleaning brush, motor, motor-driven brush, along the central axis of rotation of the blade preliminary wash, after cleaning the cleaning cloth around again wipe blade, blade cleaning completion.

Development Process to Solve Difficult Problems. To make the fan blade cleaning personnel freed from dangerous high-altitude operations, we should first solve the problem is to make the blade cleaning robot capable of independently blade surface. To solve this problem, we discuss the following work forms:

Assumption that the robot is running, the rotation of the impeller blades to be cleaned in a horizontal state, the leading edge of the blade is facing up, the robot along the leading edge, run back and forth between the root and tip, through the lower guide rail cleaning apparatus for cleaning the surface of the blade into the job. Considering the blade angle and twist itself the leading edge portion of the small size of the tip, and can not guarantee accurate robot over the track along the front line, the program can not be achieved.

The pressure difference principle, the use of high-speed vacuum centrifugal fan will be pumped into a vacuum region between the fan blades and cleaning equipment, use pneumatic robot errand tightly pressed on the leaves. Role of robots is always subjected to the atmospheric pressure and adsorbed on the running surface of the blade, the static friction force of gravity and the robot resulting equilibrium.

Blade size to be cleaned very small compared to the size of the robot, the robot we need to complete the cleaning blade coming upstream operations. Uneven blade surface, and has a certain curvature, the conventional scroll wheel does not apply. In order to produce a sufficient static friction, we chose the crawler wheel, it leaves a larger contact area and can automatically adapt to the uneven surface thereof. Tracked by a motor wheel rotation and it is mobile cleaning robot.

In addition, the blade size robotic There is a limit, when the robot to walk to the edge position, if it continues to move forward, it will happen from the blade crash, it is insecurity. To avoid this situation, we use a sensor mounted on the robot four corners. Sensor having a contact surface with the ups and downs of automatic retractable function, when the robot moves to the blade edge, the outermost sensor is floating, robotic system analysis that there is no face in a forward direction, the operation of the robot through the program back to a safe location.

(1) Brush Motor Control: Rotation of the brush motor is used to provide the desired friction cleaning blade, and since the surface material to be cleaned glass fibers, the brush bristles have a certain hardness, it does not use a lot of motor speed, while the use of a torque larger motor. We chose JGB37-520 motor, and connect it to the PLC to control it. 
If the motor is connected to a separate PLC digital output port, you can control the motor on and off. Consider cleaning efforts to get the motor to provide not necessarily what we want, it is to change the motor is connected to the analog output port on the PLC via analog output port PLC different amount of current to obtain different speeds, change the brush friction with the cleaning surface, to obtain different cleaning efforts, and set up two additional buttons access PLC, to control the current PLC analog output port for manual control of motor speed to achieve the desired cleaning intensity.

(2) Electric valve control: Electric valves provide flow control for detergent effect, the valves connected to the PLC by the PLC to perform specific control valve.

That is because the electric valve is energized rotary valve, within seconds fully open, then we will receive the valve PLC, by energizing the valve open time to control the size of the valve to control the detergent out of speed. However, due to the different needs of the cleaning surface cleaner flow rate, and when the detergent out of a certain amount of time, due to insufficient fluid pressure, the valve opening degree when using the same time, the flow rate of the cleaning agent will be gradually reduced, then we Also set the valve manually adjust buttons to fine-tune the degree of opening of the valve, thereby obtaining a suitable cleaning agent flow rate.

Final Design of the Device Structure. According to the initial plan of fan blades make intelligent cleaning robots are still some shortcomings, for some of the problems in the test, we designed the final structure of the program means to address problems early means in practical applications, so that the cleaning robot can be more effectively applied to the cleaning fan blade.

Adsorption of cleaning, walking and cleaning works consistent with the initial program. But there are different degrees of structural adjustment, schematic adjusted as follows:
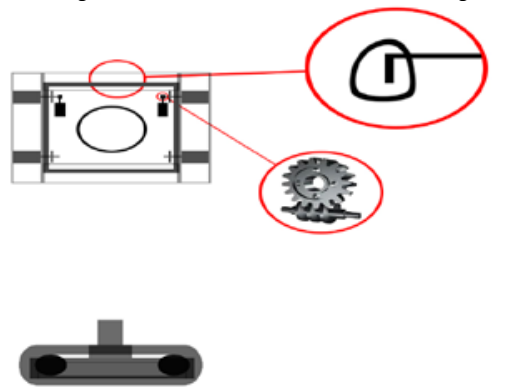

(1) Increase the power of high-speed vacuum centrifugal fan, to provide greater suction force;

(2) Increasing the area of track wheels, track wheels change material, making it suitable for uneven surface or a surface of a certain arc;

(3) Changing the way cleaning brush, increased cleaning efforts.

\section{Conclusion}

Up to today, we have completed the initial stage of the cleaning device laboratory design and production. The cleaning device has achieved an intelligent self-cleaning fan blades purposes. When connected to $220 \mathrm{~V}$ power, under the control of the PLC, the device can automatically adsorbed on the surface of the fan blade, and can be cleaned while moving work. Since the motion path of the device has not restricted the fixed model of fan blade shapes and sizes, and can be of different materials on the surface of movement, so use a wider range of the device, suitable for a variety of materials relatively flat surface cleaning, with broad development space and optimistic about the market outlook.

In future, we will further improve and refine the device. We will try to use more speed centrifugal fan, to enhance the adsorption capacity of the washer, thereby increasing the load-bearing capacity washer; or changing the material or construction crawler wheel to adapt to the different surface cleaning work. On this basis, we will increase the number of brush, accelerate the speed or change the brush cleaning method to increase the cleaning efforts. In adjustment to cleaner line, we will try to fit more models programmed fan blades clean path to achieve the whole process of automatic cleaning blade, thus greatly reducing human input. At present, our cleaning device 
more cleaning work for relatively flat surface, we will consult more literature in the late work, improve and perfect the construction of the device, making it equally applicable to a large curvature of the surface.

After the completion of the laboratory stage design and production of the model, we will be in wind farm again record the relevant data and test production of a certain size through the cleaning device and enter the trial phase. Identify and solve problems in the test run, to prepare for the full market.

\section{References}

[1] Harrison, L., Et Al., The Indicator, "Window Er Mo Nthly", Vol. 14, No. 4, April, 1998, Denmark.

[2] Zhu-Rui Z, Xue Truss, China Wind Energy Division, China Wind Papers, 108, IWC Academic Press, 1992,Beijing.

[3] Gipe, P., "Wind Energ Y Comes Of Ag E", John Wiley \& So Ns, Inc., 1995, U SA.

[4] Shi-Da Z, In '95 Beijing International Wind Energy Conference Opening Ceremony Welcoming Speech, "Wind Power", 1995 3, Beijing. 